\title{
Front Matter: Volume 10947
}

, "Front Matter: Volume 10947," Proc. SPIE 10947, Next-Generation Optical Communication: Components, Sub-Systems, and Systems VIII, 1094701 (3 June 2019); doi: 10.1117/12.2526629

SPIE. Event: SPIE OPTO, 2019, San Francisco, California, United States 


\title{
PROCEEDINGS OF SPIE
}

\section{Next-Generation Optical Communication: Components, Sub-Systems, and Systems VIII}

\author{
Guifang Li \\ Xiang Zhou \\ Editors
}

4, 6-7 February 2019

San Francisco, California, United States

Sponsored by

SPIE

Cosponsored by

Corning Incorporated (United States)

NTT Electronics (Japan)

Published by

SPIE 
The papers in this volume were part of the technical conference cited on the cover and title page. Papers were selected and subject to review by the editors and conference program committee. Some conference presentations may not be available for publication. Additional papers and presentation recordings may be available online in the SPIE Digital Library at SPIEDigitallibrary.org.

The papers reflect the work and thoughts of the authors and are published herein as submitted. The publisher is not responsible for the validity of the information or for any outcomes resulting from reliance thereon.

Please use the following format to cite material from these proceedings:

Author(s), "Title of Paper," in Next-Generation Optical Communication: Components, Sub-Systems, and Systems VIII, edited by Guifang Li, Xiang Zhou, Proceedings of SPIE Vol. 10947 (SPIE, Bellingham, WA, 2019) Seven-digit Article CID Number.

ISSN: 0277-786X

ISSN: 1996-756X (electronic)

ISBN: 9781510625365

ISBN: 9781510625372 (electronic)

Published by

SPIE

P.O. Box 10, Bellingham, Washington 98227-0010 USA

Telephone +1360 676 3290 (Pacific Time) · Fax +1 3606471445

SPIE.org

Copyright @ 2019, Society of Photo-Optical Instrumentation Engineers.

Copying of material in this book for internal or personal use, or for the internal or personal use of specific clients, beyond the fair use provisions granted by the U.S. Copyright Law is authorized by SPIE subject to payment of copying fees. The Transactional Reporting Service base fee for this volume is $\$ 18.00$ per article (or portion thereof), which should be paid directly to the Copyright Clearance Center (CCC), 222 Rosewood Drive, Danvers, MA 01923. Payment may also be made electronically through CCC Online at copyright.com. Other copying for republication, resale, advertising or promotion, or any form of systematic or multiple reproduction of any material in this book is prohibited except with permission in writing from the publisher. The CCC fee code is 0277$786 \times / 19 / \$ 18.00$.

Printed in the United States of America by Curran Associates, Inc., under license from SPIE.

Publication of record for individual papers is online in the SPIE Digital Library.

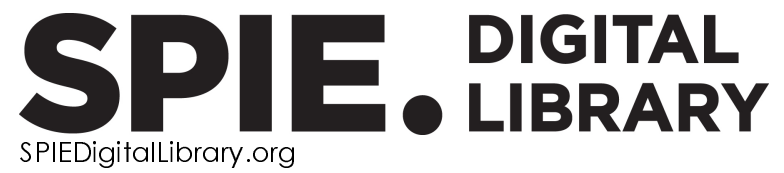

Paper Numbering: Proceedings of SPIE follow an e-First publication model. A unique citation identifier (CID) number is assigned to each article at the time of publication. Utilization of CIDs allows articles to be fully citable as soon as they are published online, and connects the same identifier to all online and print versions of the publication. SPIE uses a seven-digit CID article numbering system structured as follows:

- The first five digits correspond to the SPIE volume number.

- The last two digits indicate publication order within the volume using a Base 36 numbering system employing both numerals and letters. These two-number sets start with $00,01,02,03,04$, 05, 06, 07, 08, 09, OA, OB ... 0Z, followed by 10-1Z, 20-2Z, etc. The CID Number appears on each page of the manuscript. 


\title{
Contents
}

\author{
$\checkmark \quad$ Authors \\ vii Conference Committee
}

NOVEL SDM COMPONENTS AND TECHNIQUES

1094702 Time-dependence of the transmission matrix of a specialty few-mode fiber [10947-2]

$1094703 \quad$ Multicore fiber transmission system for high-capacity optical transport network (Invited Paper) [10947-3]

$1094704 \quad$ Photonic quasicrystal fiber supporting orbital angular momentum modes [10947-4]

1094707 Turbulence-resistant free-space communication using few-mode pre-amplifiers [10947-7]

ADVANCED MODULATION FORMAT AND PULSE SHAPING

1094708 Constellation shaping for high symbol rate SNR limited transceivers (Invited Paper) [10947-8]

10947 OA Multi-dimensional modulation and demodulation techniques for high-capacity optical transport network (Invited Paper) [10947-10]

10947 OB Numerical investigation on spatial coherent matched detection for detection of spatially multiplexed signals [10947-11]

MACHINE LEARNING APPLICATION AND OPTICS STORAGE

10947 OC Deep-learning neural network for MIMO detection in a mode-division multiplexed optical transmission system [10947-12]

10947 OD Design and optimization of quantum well electro-absorptive modulators using machine learning [10947-14]

10947 OE Lyteloop data "storage in motion" using high-bandwidth laser communications [10947-15] 
VISIBLE AND MILLIMETER-WAVE COMMUNICATION

10947 OF Connected cars: road-to-vehicle communication through visible light [10947-16]

10947 OG Coding schemes in a communication system based on visible-light communication [10947-18]

$10947 \mathrm{OH} \quad$ High-capacity IF-over-fiber transmission technology for next-generation mobile front-haul links (Invited Paper) [10947-19]

FREQUENCY COMB AND HIGH-SPEED COMPONENTS

10947 ol Optical convolution with a rectangular frequency comb for almost ideal sampling [10947-21]

10947 OJ $\quad 12.032$ Tbit/s coherent transmission using an ultra-narrow linewidth qantum dot 34.46-GHz CBand coherent comb laser [10947-23]

10947 OK Optical Nyquist pulse train generation by non-auxiliary wavelength selective switch in communication band [10947-24]

10947 OL Recent advances in ultra-high-bandwidth coherent driver modulator (Invited Paper) [10947-25]

10947 ON Experimental demonstration of three-fold wavelength multicasting of a 64-QAM 120-Gbit/s data channel using a Kerr frequency comb and nonlinear wave mixing [10947-22] 


\section{Authors}

Numbers in the index correspond to the last two digits of the seven-digit citation identifier (CID) article numbering system used in Proceedings of SPIE. The first five digits reflect the volume number. Base 36 numbering is employed for the last two digits and indicates the order of articles within the volume. Numbers start with 00, 01, 02, 03, 04, 05, 06, 07, 08, 09, OA, OB...0Z, followed by 10-12, 20-2Z, etc.

\begin{tabular}{|c|c|}
\hline Alishahi, Fatemeh, ON & Matsushita, Asuka, OA \\
\hline Almaiman, Ahmed, ON & McManamon, P. F., OE \\
\hline Alvarado Zacarias, Juan Carlos, 07 & Meier, Janosch, $\mathrm{Ol}$ \\
\hline Amezcua Correa, Rodrigo, 07 & Misra, Arijit, Ol \\
\hline Andresen, E. R., 02 & Missey, Mark, 08 \\
\hline Bekkali, Abdelmoula, $\mathrm{OH}$ & Miyamoto, Yutaka, 03, OL \\
\hline Bianconi, Simone, OD & Mohseni, Hooman, OD \\
\hline Bigot, L., 02 & Nagatani, M., OL \\
\hline Cao, Yinwen, ON & Nakajima, Kazuhide, 03 \\
\hline Choudhary, D., OE & Nakamura, Masanori, OA, OL \\
\hline Day, Pat, 08 & Nguyen, An, 08 \\
\hline Dossou, Michel, 02 & Nishimura, Kosuke, $\mathrm{OH}$ \\
\hline Fallahpour, Ahmad, ON & Nowierski, Samantha, 08 \\
\hline Fujii, T., OL & Nunoya, N., OL \\
\hline Going, Ryan, 08 & Ogiso, Y., OL \\
\hline Hamaoka, Fukutaro, OA & Oshima, Joji, OC \\
\hline Harlev, O., OE & Ozaki, J., OL \\
\hline Hashizume, Y., OL & Pfeiffer, Martin H. P., ON \\
\hline Huang, Bin, 07 & Poole, Philip, OJ \\
\hline Ida, M., OL & Poudel, Bishal, OC \\
\hline Inove, Takashi, OK & Preußler, Stefan, ol \\
\hline Ishikawa, M., OL & Rahn, Jeff, 08 \\
\hline Ishimura, Shota, $\mathrm{OH}$ & Sakamoto, Takahide, OB \\
\hline Iwashita, Katsushi, OC & Sampson, Rachel, 07 \\
\hline Kanazawa, S., OL & Samra, Parmijit, 08 \\
\hline Kandappan, Parthiban, 08 & Schneider, Thomas, $\mathrm{Ol}$ \\
\hline Karpov, Maxim, ON & Song, Chun-Ying, OJ \\
\hline Kawanishi, Tetsuya, OB & Su, Xinzhou, 07 \\
\hline Khayam, Omer, 08 & Suzuki, Kenya, 03 \\
\hline Kim, Myunghwan, 04 & Suzuki, Masatoshi, $\mathrm{OH}$ \\
\hline Kim, Soeun, 04 & Takahashi, Koji, OK \\
\hline Kippenberg, Tobias J., ON & Tanaka, Kazuki, OH \\
\hline Kisaka, Yoshiaki, OA & Tandjè, A., 02 \\
\hline Kish, Fred, 08 & Tanobe, H., OL \\
\hline Kobayashi, Hirokazu, OC & Torbatian, Mehdi, 08 \\
\hline Kobayashi, T., OL & Tremblay, Stephanie, 08 \\
\hline Koenig, Swen, 08 & Tur, Moshe, ON \\
\hline Konishi, Tsuyoshi, OK & Ueda, Y., OL \\
\hline Kordts, Arne, ON & Umezawa, Toshimasa, OB \\
\hline Le Liepvre, Alban, 08 & Vedadi, A., OE \\
\hline Lee, Chung Ghiu, 04 & Vieira, M. A., OF, OG \\
\hline Li, Guifang, 07 & Vieira, M., OF, OG \\
\hline Liao, Peicheng, ON & Vieira, P., OF \\
\hline Liu, Huiyuan, 07 & Wakita, H., OL \\
\hline Liu, Jiaren, OJ & Wang, Zhenxing, 08 \\
\hline Louro, P., OF, OG & Weber, John, OJ \\
\hline Lu, Zhenguo, OJ & Wheaton, Skyler, OD \\
\hline Maher, Robert, 08 & Willner, A. E., OE, ON \\
\hline Mao, Linda, OJ & Willner, A. N., ON \\
\hline
\end{tabular}


Wolf, Stefan, 08

Xie, Xiaojun, 08

Yamamoto, Naokatsu, OB

Yamasaki, Yu, OK

Yamazaki, H., OL

Yammine, J., 02

Zhan, Tao, 07

Ziari, Mehrdad, 08

Zou, Kaiheng, ON

Proc. of SPIE Vol. 10947 1094701-6

Downloaded From: https://www.spiedigitallibrary.org/conference-proceedings-of-spie on 26 Apr 2023 Terms of Use: https://www.spiedigitallibrary.org/terms-of-use 


\section{Conference Committee}

Symposium Chairs

Connie J. Chang-Hasnain, University of California, Berkeley (United States)

Graham T. Reed, Optoelectronics Research Centre, University of Southampton (United Kingdom)

Symposium Co-chairs

Sailing He, KTH Royal Institute of Technology (Sweden) and Zhejiang University (China)

Yasuhiro Koike, Keio University (Japan)

Program Track Chair

Benjamin B. Dingel, Nasfine Photonics, Inc. (United States)

Conference Chairs

Guifang Li, CREOL, The College of Optics and Photonics, University of Central Florida (United States)

Xiang Zhou, Google (United States)

Conference Program Committee

Kazi S. Abedin, OFS Fitel LLC (United States)

Jin-Xing Cai, TE SubCom (United States)

Hwan Seok Chung, Electronics and Telecommunications Research Institute (Korea, Republic of)

Benjamin B. Dingel, Nasfine Photonics, Inc. (United States)

Ezra Ip, NEC Laboratories America, Inc. (United States)

Yongmin Jung, University of Southampton (United Kingdom)

Inuk Kang, NOKIA Bell Laboratories (United States)

Tsuyoshi Konishi, Osaka University (Japan)

Ming-Jun Li, Corning Incorporated (United States)

Chao Lu, The Hong Kong Polytechnic University (Hong Kong, China)

Akihiro Maruta, Osaka University (Japan)

Takashi Sasaki, Innovation Core SEl, Inc. (United States)

Siyuan Yu, University of Bristol (United Kingdom)

Yanjun Zhu, Huawei Technologies Co., Ltd. (United States) 


\section{Session Chairs}

1 Optical Communication Keynote Session: Joint Session with Conferences 10945, 10946, and 10947

Benjamin B. Dingel, Nasfine Photonics, Inc. (United States)

2 Novel SDM Components and Techniques

Yutaka Miyamoto, NTT Network Innovation Laboratories (Japan)

3 Advanced Modulation Format and Pulse Shaping

An Li, FutureWei Technologies, Inc. (United States)

4 Machine Learning Application and Optics Storage

Masanori Nakamura, NTT Network Innovation Laboratories (Japan)

5 Visible and Millimeter-Wave Communication

Nicolas K. Fontaine, Nokia Bell Laboratories (United States)

6 Frequency Comb and High-Speed Components

Robert Maher, Infinera Corporation (United States) 